\title{
Merging Structural Biology with Chemical Biology: Structural Chemistry at Eskitis
}

\author{
Andreas Hofmann ${ }^{1}$, Conan K Wang ${ }^{1}$, Asiah Osman ${ }^{1}$ and David Camp ${ }^{2}$
}

(1) Structural Chemistry Program, Eskitis Institute for Cell and Molecular Therapies, Griffith University

(2) Queensland Compound Library, Eskitis Institute for Cell and Molecular Therapies, Griffith University

Corresponding author: Andreas Hofmann

\author{
Structural Chemistry Program \\ Eskitis Institute for Cell and Molecular Therapies, Griffith University \\ N75 Don Young Road \\ Nathan, Qld 4111, Australia \\ email: $\underline{\text { a.hofmann@griffith.edu.au }}$
}

Manuscript: Structural Chemistry, 8. Nov. 2010; 10019 words

Keywords: molecular mechanisms, protein crystallography, protein structure-function, rational drug design 



\begin{abstract}
This review introduces the Structural Chemistry Program at Griffith University's Eskitis Institute, and provides a brief overview over its current and future research portfolio. Capitalising on the co-location with a unique library collection of small molecules, the Queensland Compound Library, our laboratory investigates the structure and function of proteins with the aim of learning about their molecular mechanisms. Consequently, these studies also feed into drug discovery and design.

The thematic focus of our Program is on proteins involved in infection, inflammation and neurological diseases, and this review highlights a few of our recent research efforts in this area.
\end{abstract}

\title{
1 The Eskitis Institute at Griffith University
}

Research at the Eskitis Institute, directed by Prof Ronald J Quinn, investigates novel drug- and cell-based therapies for human diseases. The Institute consists of ten research groups headed by research-only Chief Investigators, and two Faculty groups who undertake research and teaching. The research groups work within in five thematic areas: Adult Stem Cell Biology, Neglected Diseases, Infection and Immunity, Cancer and Neurological Diseases. Research at the Institute, as well as the performance of individual groups, is regularly reviewed by the Scientific Advisory Board which includes three external members (Prof Chris Ireland, Dr George Moore, Dr Patrick Tan).

The laboratories and a suite of world-class facilities are housed in two co-located buildings at Brisbane Innovation Park in close neighbourhood to Griffith University's Nathan Campus. An investment of AU\$ $12 \mathrm{~m}$ in the University and the Institute by the Queensland Government enabled construction of the second of the two buildings which commenced in late 2006 and became fully operational in April 2008.

\section{About the Structural Chemistry Program}

Our Program was established in 2006 as one of ten research programs of the newly formed Eskitis Institute. Our laboratory complements the research portfolio of the Institute by providing structural information in order to understand protein mechanisms at the molecular level. To improve the knowledge of the molecular mechanisms of diseases as well as to identify possible sites of interference, one needs to have a solid understanding of the structure and functions of proteins, and their interactions with ligands, target proteins and membranes.

With protein crystallography on the one hand and a variety of biophysical methods on the other, our laboratory investigates structure, shape and properties of proteins and establishes structure-function relationships to obtain a rationale for future therapeutic applications. This includes the investigation of protein-ligand complexes as part of the drug discovery and drug design process.

Thematically, the Structural Chemistry Program focuses on two biological themes: proteins involved in (i) infection and inflammation, and (ii) neurological diseases. At protein level, our thematic focus is on cytoskeletal and membrane-associated proteins.

Since the majority of proteins we investigate are made in-house, we employ a broad range of methods from molecular cloning, protein expression and purification, providing the basis for the "wet" part of structural 
studies. Following the usual biochemical validation of purified and concentrated protein samples (SDSPAGE, UV spectroscopy, mass spectrometry), the samples are subjected to structural investigation. The core method of our laboratory is protein X-ray crystallography, and diffraction experiments are carried out at the in-house diffractometer (Rigaku Micro Max-007 generator, R-Axis IV++ detector), as well as synchrotron beam lines. Protein single crystals are obtained by screening our extensive collection $(>1000)$ of crystallisation factorials using the sitting or hanging drop vapour diffusion technique. For investigation of protein molecular mechanisms and quaternary structure, we employ biophysical methods such as circular dichroism and fluorescence spectroscopy, multi-angle light scattering, X-ray and neutron scattering, isothermal titration calorimetry, membrane and monolayer adsorption.

Since computational tasks are a major part of the structural investigation of proteins, the development of in-house software is an almost integral part of structural biology laboratories. Starting in 2002, when very few algorithms in this context were available in Java, we have been developing Java software applications for particular tasks with the aim of (i) establishing fundamental Java classes for basic operations in structural biology, and (ii) generating user-friendly, intuitive applications with graphical user interfaces for particular tasks arising in structural biology and biophysical chemistry [1; 2]. Since those days, efforts such as BioJava have grown significantly, and Java applications have now become an established part of structural biology computation. The applications developed by our laboratory are freely available for academic usage and can be downloaded from our home page (http://www.structuralchemistry.org/pcsb).

The unique collection of small molecules at the Queensland Compound Library, consisting of natural and synthetic compounds, provides a valuable resource for structural investigation of proteins with probes as well as focused drug discovery programs (Figure 1).

\section{Small molecule ligands for proteins - irreplaceable tools for drug discovery and chemical biology}

Chemical biology examines biological systems through the application of chemical techniques and tools; its goal is to use small-molecule probes to discover specific biomolecular targets and pathways that are modulated by the particular compound [3]. Small molecules, in contrast to classical genetics where manipulation occurs at the DNA level, typically modulate protein function by inducing conformational changes or by competing for endogenous protein-ligand or protein-protein interaction sites, resulting in altered activity [4]. This allows temporal study of signalling pathways and the ability to wash-out probes to study reversible inhibition.

Clearly, success derived from the innovative use of small-molecule techniques and tools depends on the creative interaction between chemistry and biology. Currently, biomedical research in Australia relies heavily on molecular biology techniques to identify biological targets and build understanding of the biological system responsible for a particular disease. These techniques allow investigators to eliminate specific proteins by 'knocking out' genes; increasing the concentrations of particular proteins by increasing the number of copies of the corresponding genes or by using a more active gene promoter; or altering the function of a protein by introducing specific mutations in the corresponding gene [5]. Although these methods are powerful in model organisms such as Saccharomyces cerevisiae and Drosophila melanogaster, mammals represent a significant experimental challenge to molecular biology approaches because of slower rates of reproduction, large sizes and large genomes. The chemical biology approach avoids these 
problems by studying the effect of small molecules on the mammalian proteome rather than the genome [5]. The Queensland Compound Library, described in more detail below, aims to facilitate biomedical research beyond classical molecular biology techniques and into new interdisciplinary sciences like chemical biology or chemical genetics, both of which require access to large diverse compound libraries.

\section{Molecular libraries}

Small molecules are critical tools for understanding important cellular events and biological pathways involved in health and disease. Access to large, diverse and biologically relevant small-molecule compound libraries is essential to the advancement of knowledge in the era of the "-omics" sciences for interrogation of biological systems (chemical biology), and the chemical optimisation of promising small-molecules into starting points (lead molecules) for the early (pre-clinical) phase of drug discovery.

The identification of new structural classes is one of the many drivers en route to innovative, safer therapeutics with novel modes of action. This is a truly daunting task given there are an estimated $10^{60}$ drug-like molecules with a molecular weight below 500 Da comprised of the atoms that make up current 'small molecule' therapies [6]. Indeed, it would be impossible to synthesize even one molecule of each member from this set considering there is estimated to be 'only' $10^{50}$ atoms on Earth [7]. Various strategies that attempt to address the relationship between chemistry and biology space have been developed in an effort to meet this grand challenge.

One such strategy has been to compile and then screen large structurally diverse libraries that number many thousands to millions of compounds against isolated biomolecular targets (usually recombinant proteins), cell-based assays and whole organisms. The development of high-throughput screening (HTS) in the late 1980s helped achieve the goal. This was soon followed by the introduction of combinatorial chemistry in the early 1990s. These synergistic technologies, coupled with advances in genomics, revolutionised drug discovery.

\subsection{Drug-like and lead-like compounds}

Combinatorial chemistry was attractive given its perceived promise to deliver large numbers of novel compounds in an effort to discover new chemical entities more efficiently. However, the early combinatorial chemistry libraries failed to live up to the hyperbole. Various reasons have been cited for the decline in new chemical entities recently including a move away from natural products that figured prominently in past paradigms [8]. However, a seminal analysis undertaken by Lipinski [9] identified that many combinatorial libraries were simply not comprised of "drug-like" molecules leading to the so called "Rule of 5 " that identified four simple physicochemical parameter ranges - molecular mass $<500$; Log $\mathrm{P}<5$; number of $\mathrm{H}$ bond donors $<5$ and; number of $\mathrm{H}$-bond acceptors $<10$ that were associated with $90 \%$ of orally active drugs achieving phase II clinical status. Lipinski's Rule of 5 is effectively a set of guidelines that identify key properties for orally administered drugs while, at the same time, highlighting potential bio-availability issues if two or more violations occur.

Much emphasis is now placed on addressing the physicochemical properties of small molecules early in the current drug discovery paradigm dominated by high-throughput screening [10]. Oprea, for example, advocated screening libraries comprised of "lead-like" or "reduced complexity" sets with even more stringent physicochemical profiles than the Rule of 5 [11; 12]. Leads are smaller and less hydrophobic than drugs. Lead-like libraries were developed in response to the generally observed trend within the 
pharmaceutical industry that a compound's properties tend to change in a consistent manner as it progresses from a lead to an approved drug. Starting below the drug-like profile identified by Lipinski allows additional mass and functionality to be introduced without potentially sacrificing oral availability.

In a somewhat more lateral approach to front loading libraries with desirable physicochemical profiles, Leeson questioned which of these properties were most important for drugs [13]. He proposed that the physicochemical parameters that remained consistent over time were the most important to consider when constructing a screening library or undertaking downstream optimisation of leads. Examination of drug approvals prior to, and after, 1983 indicated that the median Log P, percent polar surface area (\%PSA) and the number of $\mathrm{H}$-bond donors were virtually unaffected while other physico-chemical properties like the molecular mass increased steadily over time in a statistically significant manner [13]. Of the three most constant physicochemical properties identified by Leeson, Log P is considered the "Lord of the rules" for drug discovery and development [14].

\subsection{Probe compounds}

At the other extreme, a compound library could be comprised entirely of "probe" compounds or chemical "tools" [15]. Semantically, probes and tools are molecules in which the rules for lead- and drug-likeness are relaxed as the ultimate goal is not drug discovery but rather chemical biology. Basic research in the context of biopharmaceutical sciences encompasses activities concerned mainly with understanding biological and physico-chemical processes, irrespective of whether this may eventually yield a therapeutic application. In this respect, probes are chemical tools used to validate targets and interrogate function to further our understanding of biological processes.

The important point to note is that although drug discovery and probing biological function are equally important, the types of compounds used in drug discovery are more limited [15]. Target validation and interrogating biological function are not limited to molecules obeying lead- and drug-like rules. However, the two goals are not necessarily exclusive. A hit from a library based on natural products probes that do not comply with Lipinski's Rule of 5 may provide a foundation for more focused libraries that explore structure-function relationships within a particular protein family. Ultimately, enough information may be gained to progress the original chemical starting point into a molecule with a therapeutic application.

\subsection{Focused, targeted and privileged structure libraries}

Screening diverse libraries is ideal when little is known about the function or the binding of the biological target. However, because screening a large library can be cost prohibitive, there has been a shift towards focused (also called biased or directed) libraries. Library members are enriched for desired properties such as binding affinity towards the biological target [16]. As the name suggests, they are focused around a limited number of scaffolds for which knowledge of the binding interactions with a specific target is generally known [17]. Peripheral functionality is added, removed or modified in some way in an effort to improve affinity and selectivity. Targeted libraries extend the concept of focused libraries from specific chemotypes for individual targets to the generic design of libraries for target clusters and families [18]. This approach relies heavily on a variety of computational techniques [19].

Another popular strategy, especially within the pharmaceutical industry, has been to exploit 'privileged' structures with known drug-like properties. Privileged structure screening essentially relies on chemotypes such as benzodiazepines or opioids that are known to target specific gene families [20]. Conceptually, the privileged structure approach lies somewhere between focused and targeted library strategies, i.e. modifications on a known motif (focused approach) are undertaken to increase the library size for 
subsequent biological evaluation against other targets from a gene cluster or family (targeted strategy). Interestingly, some common chemotypes are active against different gene families, indicating that diverse biological targets can contain structurally common small-molecule binding sites [10].

However, it must be kept in mind that any enhancement of screening collections using focussed, targeted and privileged library design strategies risks over-enrichment with specific structural classes that can dilute the serendipitous value of high-throughput screening techniques and endanger potential intellectual property [10; 18]. Diversity oriented synthesis [21; 22] is a relatively new approach that addresses accessible structural diversity of a library by mimicking the structural complexity and diversity of natural products [16].

\section{The Queensland Compound Library}

\subsection{Genesis and operational aspects}

Until recently a lack of critical infrastructure prohibited consolidation of the chemical output from disparate researchers in Australasia into a central small molecule repository to facilitate screening. To address this imbalance, an automated compound management facility, the Queensland Compound Library (QCL), was established at the Eskitis Institute to actively source small molecules from the current Australian pool of mainly academic researchers [23]. The consolidation of open access small molecules into a central repository has several benefits: a greater coverage of chemistry space is achieved compared to isolated collections; screening in the high-throughput screening paradigm is facilitated as the collection is sizeable and can be reformatted directly en masse into labware used by biological research teams; molecules that may otherwise have been forgotten can now potentially have value added if they are bioactive by way of publications or patents and; synergies and collaborations between chemists and biologists are encouraged [24].

As a national resource, the QCL has had to be flexible in its approach for the deposition and access to compounds given the variety of organizations that use the facility. An example of this is how chemists can utilize their samples. Passive storage occurs when the chemist submits samples for potential access by biologists. In this mode the chemist essentially allows their molecules to be used by interested biologists on an ad hoc basis. Proactive storage, on the other hand, is where the chemist pursues third party collaborations. This mechanism significantly increases the likelihood of a chemist's collection being the starting point of downstream projects.

Another example of the flexibility adopted by the $\mathrm{QCL}$ is the handling of intellectual property (IP). The QCL does not lay claim to any IP owned or generated by users of the facility. The QCL model allows synergies to develop and mature into projects that are prosecuted in a way best suited to the collaboration. Thus, molecules submitted by chemists may be tested to interrogate biological function or form the basis of a drug discovery program. In essence, the $\mathrm{QCL}$ is a facility that encourages collaborative research.

\subsection{Infrastructure}

The QCL was established using three main building blocks: a microtube store; a sample processing unit and; a microtitre plate store (Figure 2). Environmentally controlled plate and microtube storage is integrated with sample processing to ensure that compound integrity is maintained without compromising flexibility. 
Modular components that allowed a stepwise increase in capacity or throughput if required were selected. The initial configuration comprised two microtube stores, each capable of storing 100,000 microtubes, a plate store (with a capacity of 2,160 standard microtitre plates), a plate server and a sample processing unit. Since 2008, an additional two microtube stores and one plate store have been added.

Stock solutions are prepared in microtubes, each of which is identified by an individual 2D barcode which is read on the way into and out of the stores to assist cradle-to-grave tracking of all samples. Requested samples are individually picked from the microtube store, obviating unnecessary freeze-thaw cycles of additional samples intrinsic to plate-based systems. Moreover, racks of retrieved tubes can be arrayed in user-specified formats to simplify subsequent plate creation. Microtubes are typically reformatted into 'working' plates, which are subsequently replicated into assay ready plates using $\mu \mathrm{L}$ and $\mathrm{nL}$ liquid transfer equipment located within the sample processing unit.

\subsection{Synergies between molecular libraries and structural chemistry programs}

The combination of chemistry resources, chemical biology, structural chemistry and biological screening at the Eskitis Institute has aided the identification of several new leads for neglected diseases like malaria and human African trypanomiasis [25-29], as well as drug discovery for carbonic anhydrases [30]. Further work, as described below utilises structural chemistry techniques to gain insights into neglected diseases using small molecules in chemical biology projects. Many of the molecules sourced by the Structural Chemistry Program originate from the Eskitis Molecular Libraries Initiative (EMLi). Here, the natural product and synthetic chemists have achieved, at the Institute level, what the QCL aims to do at the national level. The chemistry is combined into the EMLi and used as requested. Over 2,500 unique small molecules derived predominately from natural products research can be accessed by the Structural Chemistry laboratory for further study. The Structural Chemistry Program is also a part of a larger cancer drug discovery effort that spans several Australian universities, medical research institutes and other international partners. Here, 150,000 compounds owned by the overarching organisation are stored at the $\mathrm{QCL}$ and can be used to gain insights into ligand-receptor binding.

\section{Research Portfolio}

\subsection{Structural Chemistry of Proteins involved in Infection and Inflammation}

\subsubsection{Parasite Proteins}

Parasitism is a major burden for humans and livestock world-wide. Many neglected tropical diseases afflicting scores of the world's poorest peoples are caused by parasites. Little scientific or drug discovery work has been done on many of these aptly named "neglected" diseases, and, importantly, there are serious problems with resistance in nematodes against all of the main classes of anti-parasitic compounds that have been used for the past $40+$ years to treat the diseases they cause [31]. For the future, it is expected that the changing climate will lead to an expansion of parasitism into areas that are currently unaffected, and, as a consequence, the prevalence of parasitic diseases is expected to rise. The rapidly growing literature on parasites in arctic and subarctic ecosystems indicates that climate-linked changes have already occurred [32].

While the amenability of parasite enzymes for drug intervention provides an immediate argument for their importance in this context, recent studies have also put non-enzymatic proteins responsible for hostpathogen interactions into focus in the search for new therapeutics. The abundance and common presence of some proteins (activation-associated secreted proteins; ASPs) in excretory/secretory (ES) products of 
parasitic nematodes may present a way of intervention for an entire range of different parasitic species [33]. In other organisms, such as Schistosomes or Giardia, conserved proteins supporting the integrity of the parasite are generally believed to constitute virulence factors and thus also are attractive non-enzymatic targets.

\subsubsection{Parasite-specific enzymes}

Phosphorylating and dephosphorylating enzymes are a group of proteins essential for the regulation of a large variety of cellular processes, including cell-cycle progression, transcription, DNA replication and metabolic functions [34]. Serine/threonine phosphatases (STPs) are usually cytoplasmic proteins and often involved in signal transduction and transcriptional activation [35]. Male-specific STPs have recently been identified from the pork parasite Oesophagostomum dentatum and the parasite Trichostrongylus vitrinus [36], a pathogen affecting mainly lambs, rabbits and goats, as well as from Haemonchus contortus, a strongylid nematode parasite of small ruminants [37]. Our structural analyses of the proteins, by comparative modelling, confirmed higher similarity of these STPs to protein phosphatase 1 (PP1)-type STPs like glc seven-like phosphatase GSP-3 and -4 , which appear to be nematode specific, than other PP1 STPS such as GSP-1 and -2, which are highly conserved amongst nematodes and other organisms such as mammals [37]. Since the functions of proteins GSP-3 and GSP-4 are known to be central to spermatogenesis and other male-specific processes in Caenorhabditis elegans, the newly identified nematode STPs may play a significant role in reproductive and/or developmental processes in maturing or adult male parasites, and thus there is prospect for novel nematocides [38].

\subsubsection{Proteins involved in host-pathogen interactions}

The richly diverse morphological, behavioural and developmental alterations in parasites are matched by the evolution of distinctive and characteristic protein families [39]. Most often, however, the molecules expressed by parasitic groups have high sequence identity with those expressed by their free-living relatives, or their hosts. How these ubiquitous molecules are used in parasitism is a question of major interest.

Activation-associated secreted proteins (ASPs) have been the focus of attention in blood-feeding nematodes, as they have been shown to induce protective immune responses (reviewed by [40]). ASPs are members of the SCP/TAPS family of proteins, and while the functions of most of these proteins are largely unknown, recent advances in genome sequencing and proteomics have revealed a massive expansion of this gene family in parasitic helminths, particularly in the blood-feeding hookworms. The only hookworm SCP/TAPS protein for which a function has been shown is the neutrophil inhibitory factor (NIF), a protein that binds to the CR3 receptor integrin and inhibits neutrophil recruitment [41], supporting an immunomodulatory role for at least some hookworm ASPs.

From the structure-based amino acid sequence alignment, using the crystal structure of Na-ASP-2 from Necator americanus [42] as a template, we conclude that the general fold is likely to be conserved throughout the family of ASPs. This conclusion is also supported by the structures of the major venom 
allergen of yellow jackets, Ves v5 [43] and the NMR structure of P14a from plants [44]. Comparative modelling studies (Winter, Hofmann, Loukas \& Gasser, unpublished) have demonstrated that there are at least three groups of ASPs (Groups 1-3), and we have made three main observations: (i) While the ASPs from Groups 1 and 3 adopt similar, globular shapes, the representative structure of Group 2 indicates a more elongated shape, due to the lack of dithioether linkages and subsequent unfolding of a loop segment. The extended shape agrees with preliminary results from hydrodynamic experiments (Hofmann \& Loukas, unpublished data); (ii) ASPs possess an equatorial groove around the protein (Figure 3). This groove is most prominent for molecules in Groups 1, and is proposed to serve as binding site for 'sequential' ligands, such as peptides. The different electrostatic properties of the representative structures for molecules in Groups 1 and 3 are hypothesised to provide the molecular basis for ligand specificity; (iii) ASPs in Group 1 possess a putative catalytic site. Recently, we specifically identified a putative catalytic site formed by residues His69 and His129 (with potentially activating residues in the periphery) (Hofmann \& Loukas, unpublished). This arrangement is reminiscent of that observed for other enzymes (e.g. cyclic nucleotide phosphodiesterases) [45]. To discover the largely unknown features of ASPs and their potential for therapeutic strategies is the subject of ongoing studies in our Program.

Annexins are soluble proteins with the hallmark feature of calcium-dependent binding to phospholipid membranes. Annexins are widely expressed in multicellular eukaryotes as well as prokaryotes. While annexin proteins have been identified in the model nematode Caenorhabditis elegans and the pathogenic protozoan Giardia some time ago, worm and parasite annexins started to receive more attention only recently. The completion of the genomes of Schistosoma mansoni and Schistosoma japonicum revealed the presence of a subfamily of annexin proteins in these organisms. Interestingly, while annexins have been shown to exist in the Anopheles mosquito, no annexin-like protein has yet been discovered in any Plasmodium species.

Individual functions have only begun to be assigned to few members of the mammalian subfamily of these proteins. Typical annexin activities are focused on membrane-membrane interactions in vesicular transport (endo- and exocytosis), membrane attachment of the cytoskeleton. Several members of the protein family have also been implicated in extracellular functions, but again with involvement of membrane surfaces. Annexin proteins possess a distinct, highly conserved fold [46], which enables general adapter-like functions at the surface of phospholipid membranes. Underpinning this functionality, many interactions of annexins with other proteins have been observed, but to date only very few structural details are known.

Annexin proteins are characterised by a fourfold repeat (IIV) of a 70 amino acid sequence folding into a four-helix barrel (helices A, B, D and E) that is capped by a fifth $\alpha$-helix (C) perpendicular to the barrel [46]. At the top of the barrel, the connecting loops between helices $A / B$ and $D / E$ harbour binding sites for calcium ions that mediate the binding of the protein molecules to acidic phospholipid membranes. To distinguish these binding sites from the ones found in EF-hand proteins (type I), they have been termed type II and type III sites. At high concentrations of metal ions, a second metal ion can be bound in the $A B$ loop, in a site called AB' [47]. Recent structural investigation of alpha-11 and alpha-14 giardin revealed that the annexin fold also allows for fourth type of calcium coordination in a site termed type IIIb [48; 49]. Notably, annexins possess very low affinity for divalent metal ions in solution; calcium is only bound to the protein in the presence of phospholipid membranes.

From our studies with plant annexins, it became clear that there are differential membrane binding mechanisms in different sub-families of annexins [50]. Plant annexins lack the endonexin sequence in some repeats but possess conserved basic and hydrophobic residues on the convex, membrane-binding side which actively participate in interactions with the membrane $[51 ; 52]$. Preliminary studies with parasite 
annexins in our lab indicate a similar situation with that group of annexins [53](Osman \& Hofmann, unpublished).

Some mammalian annexins [54] as well as alpha giardins [55] have been reported to possess lectin properties. Following the initial idea that externalised parasite annexins may use glycosaminoglycan binding as a means of attachment to the host tissue, we have previously characterised the calcium-dependent binding of Taenia solium annexin B1 to heparin and developed a model [53] using the structural data available for annexin A5 [56]. Ongoing studies with alpha giardins and Schistosome annexins in our lab indicate that this is not a commonly shared property among parasite annexins (Osman et al., unpublished).

We have recently conducted a survey of annexins from a variety of parasites, including protistan, (inc. Giardia), and metazoans (nematodes and platyhelminths) [57]. Using secondary structure prediction, structure-based sequence alignments and comparative modelling, we have identified a unique structural feature shared by many parasite annexins, including many from Schistosomes. These proteins possess an unusually long linker between repeats II and III which is predicted to adopt $\alpha$ helical secondary structure. We thus believe that this common feature of parasite annexins, which also separates them from their mammalian relatives, is worth pursuing as an antigenic epitope in multi-constituent vaccines.

Additionally, the annexins may also be a target for drug intervention. The primary functions of annexins known to date are centred around their ability to bind ultrastructural surfaces such as phospholipid membranes and their associated proteoglycan network. Potential drugs therefore need to physically interfere in the binding sites of annexins to membranes, sugars and proteins, or alter the binding behaviour through allosteric mechanisms. We and others have previously shown that mammalian annexins possess a binding pocket for small molecules on the concave side and that different benzo-di/thi-azepines affect the annexin-mediated membrane permeabilisation to varying degrees [58; 59].

\subsubsection{Human annexin A1}

Human annexin A1 has long been of pharmaceutical interest, since it is up-regulated by anti-inflammatory glucocorticoids and also mediates cellular glucocorticoids [60]. From a structure-function perspective, annexin $\mathrm{A} 1$ has also been a focus of major interest, since it belongs to the group of mammalian annexins that promotes membrane aggregation [61], unlike annexin A5. Furthermore, in contrast to most other members of this protein family, annexin $A 1$ is able to bind to phospholipid membranes in the absence of calcium, and this interaction has been mapped to the $\mathrm{N}$-terminal domain of annexin $\mathrm{A} 1$ [62]. The molecular mechanism of annexin A1-mediated membrane aggregation is unclear, but three models have been suggested. In one scenario, two adjacent membrane surfaces are linked by one annexin molecule which simultaneously employs the canonical membrane binding mode via the convex side and the calciumindependent membrane binding mode via the $\mathrm{N}$-terminal domain. A second scenario can arise from a heterodimeric annexin A1 with an interaction interface in the $\mathrm{N}$-terminal domain, thus contacting two adjacent membrane surfaces via the convex protein surfaces. The third possibility is analogous to annexin $\mathrm{A} 2$, and assumes an annexin A1 heterotetramer held together by a binding partner, for example S100A6 or S100A11 [63]. The membrane binding mode in the last scenario would also involve the canonical binding 
sites on the convex surfaces. While the last model is certainly a possibility under physiological conditions, it cannot explain the in vitro aggregation behaviour of annexin A1 conducted in the absence of other proteins.

Using CD spectroscopy, we have shown that annexin $\mathrm{A} 1 \mathrm{~N}$-terminal peptides are unstructured in solution, but can adopt a helical fold in the presence of phospholipid membranes in the absence of calcium [64]. Under the same conditions, we have also observed binding of the peptides to phospholipid monolayers with surface film balance measurements. At the molecular level, we investigated the membrane binding of these peptides by neutron diffraction which revealed a parallel orientation of the peptides with respect to the surface of the membrane stacks. These observations therefore support a model where annexin A1 membrane aggregation arises from the simultaneous interactions of one protein molecule with adjacent membrane surfaces.

\subsection{Structural Chemistry of Proteins involved in Neurological Diseases}

\subsubsection{Visinin-like Proteins}

Neuronal calcium sensor (NCS) proteins play key roles in controlling neuronal function [65], and have been implicated in a variety of physiological functions ranging from synaptic plasticity [66; 67], neuropathological processes [68; 69], pain modulation [70] to cancer [71].

In response to elevated cytosolic calcium levels, NCS proteins such as Visinin-like Proteins (VILIPs) change their molecular conformation and re-locate to distinct sub-cellular compartments. In particular, VILIPs colocalise with intracellular membranes that contain specific phospho-inositides. Different VILIPS act as effectors to transduce different calcium signals, with VILIP-1 modulating the activities of adenylyl and guanylyl cyclases. This pivotal role in neurotransmission has implicated VILIPs in synaptic plasticity and the pathophysiology of schizophrenia, as well as calcium-mediated neurodegeneration, including Alzheimer's disease, and cancer.

There are several lines of observations supporting the notion that the differential localisation of VILIPS might be due to specific membrane binding capabilities of the individual proteins. Recently, the occurrence of polybasic and polybasic-lipoyl motifs have been correlated with the targeting of plasma membrane proteins due to recognition of $\mathrm{PI}(3,4,5) \mathrm{P}_{3}$ and $\mathrm{PI}(4,5) \mathrm{P}_{2}[72 ; 73]$. A similar observation is made with the cytosolic domain of alpha1 integrin [74], as well as VILIPs and hippocalcin [75] which possess several basic residues in their $\mathrm{N}$-terminal region. Since there is no experimental three-dimensional structure of VILIPS available so far, we have generated three-dimensional models of VILIP-1 and VILIP-3 in the apo- and the calcium-bound forms by comparative modelling, based on the high similarity of VILIPs with neurocalcin $d$ and recoverin [76; 77]. Using models with docked phospho-inositides in molecular dynamics studies, it appears that VILIP-1 and VILIP-3 are able to bind various phospho-inositide derivatives in a site located in the N-terminal area [78]. The anticipated basic amino acids play a key role in ligand binding. Interestingly, conformational flexibility of the protein, through orienting the $\mathrm{N}$-terminal region of the protein against its core (hinge between Thr20 and Glu21), seems to be a requirement for ligand binding.

Using a phospholipid overlay (PIP strip) assay, we examined binding of myristoylated VILIP-1 to various phospholipids, and found the protein to bind to phospho-inositides and phosphatidic acid, but not to phosphatidylserine, -choline, or -ethanolamine [78]. These findings are in agreement with preliminary adsorption experiments to phospholipid monolayers using unmyristoylated VILIP-1. With PS/PC (3:1) 
monolayers, VILIP-1 in the absence of calcium elicits a very slow increase of the surface pressure. With monolayers that contain either $1 \% \mathrm{PI}(4,5) \mathrm{P}_{2}$ or $\mathrm{PI}(3,4,5) \mathrm{P}_{3}$, a sudden increase in surface pressure is observed. Notably, this demonstrates that even in the absence of calcium, VILIP-1 can interact with phospho-inositides embedded in a membrane monolayer. Taking into account the structural context of the proposed PIP binding site in the N-terminal region, it appears that membrane-anchored PIP can elicit a conformational change of VILIP-1 from its "closed" (myristoyl group harboured in the protein) to an "open" (myristoyl group exposed) state that allows access to the binding site. While this is believed to happen upon binding of calcium to the protein during the calcium-myristoyl switch, the current results suggest that a similar mechanism can be triggered by membrane-anchored PIP.

We also looked at the interaction of VILIP-1 with $\mathrm{PI}(4,5) \mathrm{P}_{2}$ in hippocampal neurons, and found a clear colocalisation of VILIP-1 and $\mathrm{PI}(4,5) \mathrm{P}_{2}$ at intracellular localisations and at defined spots at the cell surface.

In a collaborative effort between the labs of the Structural Chemistry and the Molecular Libraries Program, we are currently developing molecular probes for PIP signalling and will use these as tools to obtain further details of the VILIP-PIP interactions. Small molecules are valuable tools for understanding important cellular events and biological pathways involved in health and disease. By developing novel molecular libraries to probe proteins involved in PIP signalling, we will obtain tools that provide temporal, reversible modulation of cellular systems to address the most challenging and significant problems associated with aberrant pathway activity. Typically, inositol phosphate derivatives are needed that mimic the physiologically relevant part of phospho inositides, which may or may not include the glyceryl moiety, but lack the fatty acid groups in order to avoid unfavourable effects in solution. Particularly exciting will be the generation of novel compounds that incorporate a scaffold inspired by inositol phosphate. These compounds will provide the basis for developing novel probes to interrogate PIP signalling proteins as well as chemical biology-driven drug discovery for such proteins. As an example for such novel probes, we also intend to include heavy atoms (heavy metal or halide atoms) into these small molecules which will enable experimental phasing in $\mathrm{X}$-ray crystallography experiments.

Based on experimental data on the interaction between GCAP1 and retinal GC [79], we have previously mapped residues on our VILIP-1 homology model that are putatively important for interactions with guanylyl cyclase [76]. These interactions with the membrane receptor can occur in the interfacial groove area between EF3 and EF4, as well as the VILIP-1 dimer interface in the N-terminal region. Notably, Cys187 is located in this groove between EF3 and EF4 which would enable formation of a disulphide bond between the NCS protein and the membrane receptor. This model would be in agreement with recent findings that a Cys187Ala mutant of VILIP-1 showed significantly reduced GC activation [80]. Ongoing efforts are directed at the characterisation of possible direct interactions between VILIPs and guanylyl cyclase.

\subsubsection{Coronins}

Coronins are conserved actin-binding proteins that promote processes that rely on remodelling of the actin cytoskeleton. They are characterised by a short $\mathrm{N}$-terminal stretch that contains a dodecapeptide with several basic amino acids (the coronin signature motif), followed by a WD40-repeat region forming a sevenbladed $\beta$-propeller structure [81]. The propeller domain is linked to a C-terminal extension that is unique for each member of the coronin protein family. The very $\mathrm{C}$-terminal domain in most coronins is comprised 
of a coiled coil that is responsible for homo-oligomerisation observed with several coronins.

Proteins possessing WD40-motifs play essential roles in various cellular processes like migration, signal transduction, RNA-processing, regulation of transcription, cell cycle, apoptosis, cytoskeletal dynamics, and vesicle trafficking [82-84]. It is thus not surprising that some WD40-proteins have been shown to be involved in human diseases [85]. The $\beta$-subunit of heterotrimeric G-proteins $(G \beta)$ is one of the structurally best-characterised proteins carrying a WD40-domain [86].

The WD40-motif is characterised by the dipeptide GH located 11-24 amino acid positions from the Nterminus of the repeat, and the dipeptide WD at its C-terminus; however, there is some variation as to the presence and constitution of these dipeptides in various WD40-domains [83].

Until recently, CRN2 (synonyms: coronin-1C, coronin-3), like all short coronin proteins, was thought to consist of an N-terminal domain of about 70 amino acids length, a core region containing five WD40domains, and a C-terminal domain comprising about 170 amino acids [87; 88]. Using secondary structure prediction and manual amino acid alignments, we have proposed a structure for CRN2 (synonyms: coronin1C, coronin-3) that contains the fold of a seven-bladed propeller in the central domain. We proposed that of the two 'additional' WD40-repeats one is located in the N-terminal domain, and the other in the conserved part of the C-terminal domain [89], and a similar model was put forward by Gatfield and colleagues [90]. This hypothesis was subsequently confirmed by the crystal structure of truncated CRN4 (synonyms: coronin$1 \mathrm{~A}$, coronin-1) which revealed the presence of seven WD40-repeat domains [86].

Of particular current interest is the quaternary structure of various coronins and their regulation. Current working models of coronin function suggest the presence of monomers and trimers that exert different activities such as actin or Arp2/3 complex binding [91]. Recently, we identified and characterised two novel CRN2 (synonyms: coronin-1C, coronin-3) isoforms, referred to as CRN2i2 and CRN2i3, which possess elongated $\mathrm{N}$-termini when compared to CRN2i1 [92]. Intriguingly, size exclusion chromatography revealed that the isoform with the longest $\mathrm{N}$-terminal domain, CRN2i3, exists in monomeric form, while the other two isoforms are present as trimers in solution. In the (predicted) three-dimensional structure, the $\mathrm{N}$ - and C-termini of the coronin $\beta$-propeller are spatially close, and it is thus tempting to speculate that the additional $\mathrm{N}$-terminal peptide in the case of CRN2i3 may interact with the $\mathrm{C}$-terminal coiled coil domain. These interactions may prevent the formation of trimers due to formation of three-stranded coiled coils which seems to be the case in CRN2i1 and CRN2i2. This hypothesis is supported by the fact that there are two heptapepide motifs (abcdefg) in the N-terminal extension of CRN2i3 that may form intra-molecular coiled-coil interactions with peptides in the C-terminal region, thus making it impossible for the intermolecular three-stranded coiled coil to form.

Additionally, phosphorylation could turn out to be another regulator of coronin quaternary structure. In silico analyses predict several post-translational modifications of CRN2, and, importantly, two phosphorylation sites in the C-terminal coiled coil region. Since phosphorylation has been reported to regulate the activity of coronins [93; 94], the regulation of the CRN2 quaternary structure via posttranslational modification may form an essential factor of the CRN2 activity.

\section{Conclusions}

The work flow in most protein crystallography/structural biology laboratories today has changed significantly compared to the 1990s. Software improvements and automation have allowed for faster throughput and tackling of more difficult protein systems. It is also the general scientific question that has 
shifted; in rare cases, 'only' the protein structure is being studied. Instead, structural investigation nowadays almost always involves embedding the protein structure into its biological context, either through considering protein interactions with other biological macromolecules or through probing the protein features with the help of small molecules. In both cases, valuable insights into the architectonics [95] of life are enabled, leading to an understanding of the molecular basis of health and disease, as well as feeding into drug discovery projects.

The Structural Chemistry Program through its unique embedding in the Eskitis Institute, and in particular through the close collaboration with the Queensland Compound Library as well as various expert biology laboratories, combines the protein structure theme with biological mechanisms and chemical tool sets.

\section{Acknowledgements}

Research in the Structural Chemistry Program is funded by the Australian Research Council, Griffith University, the James N Kirby Foundation, the National Health and Medical Research Council (Fellowship to CKW), and the Rebecca L Cooper Foundation. We gratefully acknowledge the Australian Synchrotron for beam time awards.

\section{References}

1. Hofmann A, Wlodawer A (2002) PCSB - a program collection for structural biology and biophysical chemistry. Bioinformatics 18: 209-210

2. Hofmann A (2008) ACDP - a Java application for data processing and analysis of protein circular dichroism spectra. J. Appl. Crystallogr. 42: 137-139

3. Dobson CM (2004) Chemical space and biology. Nature 432: $824-828$

4. Buskirk AR, Liu DR (2005) Creating small-molecule-dependent switches to modulate biological functions. Chemistry \& Biology 12: 151-161

5. Stockwell BR (2004) Exploring biology with small organic molecules. Nature 432: 846-854

6. Bohacek RS, McMartin C, Guida WC (1996) The art and practice of structure-based drug design: a molecular modeling perspective. Med Res Rev 16: 3-50

7. Spring DR (2003) Diversity-oriented synthesis; a challenge for synthetic chemists. Org Biomol Chem 1: 3867-3870

8. Newman DJ, Cragg GM (2007) Natural products as sources of new drugs over the last 25 years. J. Nat. Prod. 70: 461-477

9. Lipinski C, Lombardo F, Dominy B, Feeney P (1997) Experimental and computational approaches to estimate solubility and permeability in drug discovery and development settings. Adv. Drug 
Deliv. Rev. 23: 3-25

10. Leeson P, Davis A, Steele J (2004) Drug-like properties: guiding principles for design - or chemical prejudice?. Drug Discov Today Technol 1: 189-195

11. Teague SJ, Davis AM, Leeson PD, Oprea T (1999) The Design of Leadlike Combinatorial Libraries. Angew. Chem. Int. Ed. Engl. 38: 3743-3747

12. Hann MM, Oprea TI (2004) Pursuing the leadlikeness concept in pharmaceutical research. Curr. Opin. Chem. Biol. 8: 255-263

13. Leeson PD, Davis AM (2004) Time-related differences in the physical property profiles of oral drugs. J. Med. Chem. 47: 6338-6348

14. Ganesan A (2008) The impact of natural products upon modern drug discovery. Curr. Opin. Chem. Biol. 12: 306-317

15. Lipinski CA, Hopkins A (2004) Navigating chemical space for biology and medicine. Nature 432: 855-861

16. Bredel M, Jacoby E (2004) Chemogenomics: An emerging strategy for rapid target and drug discovery. Nat Rev Gen 5: 262-275

17. Golebiowski A, Klopfenstein SR, Portlock DE (2001) Lead compounds discovered from libraries. Curr. Opin. Chem. Biol. 5: 273-284

18. Prien O (2005) Target-Family-Oriented Focused Libraries for Kinases - Conceptual Design Aspects and Commercial Availability. ChemBioChem 6: 500-505

19. Player MR (2004) Target-based compound library design and synthesis. Drug Discov Today Targets 3: $48-50$

20. DeSimone RW, Currie KS, Mitchell SA, Darrow JW, Pippin DA (2004) Privileged Structures: Applications in Drug Discovery. Comb Chem High Throughput Screen 7: 473-493

21. Burke MD, Berger EM, Schreiber SL (2003) Generating Diverse Skeletons of Small Molecules Combinatorially. Science 302: 613-618

22. Fergus S, Bender A, Spring DR (2005) Assessment of structural diversity in combinatorial synthesis. Curr. Opin. Chem. Biol. 9: 304-309

23. Camp D (2007) Establishment of an open access compound management facility in Australia to stimulate applied, basic and translational biomedical research. Drug Discov World 8: 61-66

24. Camp D, Avery VM, Street I, Quinn RJ (2007) Progress towards establishing an open access molecular screening capability in the Australasian region. ACS Chem. Biol. 2: 764-767

25. Mueller D, Davis RA, Duffy S, Avery VM, Camp D, Quinn RJ (2009) Antimalarial Activity of Azafluorenone Alkaloids from the Australian Tree Mitrephora diversifolia. J. Nat. Prod. 72: 1538- 
26. Davis RA, Duffy S, Avery VM, Camp D, Hooper JNA, Quinn RJ (2010) (+)-7-Bromotrypargine, an Antimalarial beta-Carboline from the Australian Marine Sponge, Ancorina sp.. Tetrahedron Lett. 51: 583-585

27. Feng Y, Davis RA, Sykes ML, Avery VM, Camp D, Quinn RJ (2010) Antitrypanosomal cyclic polyketide peroxides from the Australian marine sponge Plakortis sp.. J. Nat. Prod. 73: 716-719

28. Feng Y, Davis RA, Sykes ML, Avery VM, Carroll AR, Camp D, Quinn RJ (2010) Antitrypanosomal pyridoacridine alkaloids from the Australian ascidian, Polysyncraton echinatum. Tetrahedron Lett. 51: $2477-2479$

29. Yang X, Davis RA, Buchanan MS, Duffy S, Avery VM, Camp D, Quinn RJ (2010) Antimalarial bromotyrosine derivatives from the Australian marine sponge Hyattella sp.. J. Nat. Prod. 73: 985987

30. Lopez M, Paul B, Hofmann A, Innocenti A, Vullo D, Supuran C, Poulsen S (2009) S-glycosyl primary sulfonamides - A new structural class for selective inhibition of cancer-associated carbonic anhydrases. J. Med. Chem. 52: 6421-6432

31. Besier B (2007) New anthelmintics for livestock: the time is right. Trends Parasitol. 23: 21-24

32. Kutz S, Dobson A, Hoberg E (2009) Where are the parasites?. Science 326: 1187-1188

33. Cantacessi C, Campbell BE, Visser A, Geldhof $P$, Nolan MJ, Nisbet AJ, Matthews JB, Loukas A, Hofmann A, Otranto D et al. (2009) A portrait of the "SCP/TAPS" proteins of eukaryotes-developing a framework for fundamental research and biotechnological outcomes. Biotechnol. Adv. 27: 376-388

34. Hanks SK, Quinn AM, Hunter T (1988) The protein kinase family: conserved features and deduced phylogeny of the catalytic domains. Science 241: 42-52

35. Barford D, Das A, Egloff M (1998) The structure and mechanism of protein phosphatases: insights into catalysis and regulation. Annu. Rev. Biophys. Biomol. Struct. 27: 133-164

36. Hu M, Abs El-Osta YG, Campbell BE, Boag PR, Nisbet AJ, Beveridge I, Gasser RB (2007) Trichostrongylus vitrinus (Nematoda: Strongylida): Molecular characterisation and transcriptional analysis of TV-stp-1, a serine/threonine phosphatase gene. Exp. Parasitol. 117: 22-34

37. Campbell B, Rabelo E, Hofmann A, Hu M, Gasser R (2010) Characterization of a Caenorhabditis elegans Glc seven-like phosphatase (gsp) orthologue from Haemonchus contortus (Nematoda). Mol. Cell. Probes in press:

38. Campbell, B.E., McCluskey A, Hofmann A, Gasser R (2010) Serine/threonine phosphatases in socioeconomically important parasitic nematodes - prospects as novel drug targets?. Submitted:

39. Nolan MJ, Hofmann A, Jex AR, Gasser RB (2010) A theoretical study to establish the relationship between the three-dimensional structure of triose-phosphate isomerase of Giardia 
duodenalis and point mutations in the respective gene. Mol. Cell. Probes in press:

40. Loukas A, Bethony J, Brooker S, Hotez P (2006) Hookworm vaccines: past, present, and future. Lancet Infect Dis 6: 733-741

41. Muchowski PJ, Zhang L, Chang ER, Soule HR, Plow EF, Moyle M (1994) Functional interaction between the integrin antagonist neutrophil inhibitory factor and the I domain of CD11b/CD18. J. Biol. Chem. 269: 26419-26423

42. Asojo OA, Goud G, Dhar K, Loukas A, Zhan B, Deumic V, Liu S, Borgstahl GEO, Hotez PJ (2005) Xray structure of Na-ASP-2, a pathogenesis-related-1 protein from the nematode parasite, Necator americanus, and a vaccine antigen for human hookworm infection.. J. Mol. Biol. 346: 801-814

43. Henriksen A, King TP, Mirza O, Monsalve RI, Meno K, Ipsen H, Larsen JN, Gajhede M, Spangfort MD (2001) Major venom allergen of yellow jackets, Ves v 5: structural characterization of a pathogenesis-related protein superfamily.. Proteins 45: 438-448

44. Fernández C, Szyperski T, Bruyère $T$, Ramage $P$, Mösinger $E$, Wüthrich $K$ (1997) NMR solution structure of the pathogenesis-related protein P14a.. J. Mol. Biol. 266: 576-593

45. Hofmann A, Zdanov A, Genschik P, Ruvinov S, Filipowicz W, Wlodawer A (2000) Structure and mechanism of activity of the cyclic phosphodiesterase of Appr $>p$, a product of the tRNA splicing reaction. EMBO J. 19: 6207-6217

46. Hofmann A, Huber R (2003) Structural conservation and functional versatility: allostery as a common annexin feature. In: Bandorowicz-Pikula J (ed) Annexins: biological importance and annexin-related pathologies. Landes Bioscience, Georgetown, TX.

47. Sopkova J, Renouard M, Lewit-Bentley A (1993) The crystal structure of a new high-calcium form of annexin V. J. Mol. Biol 234: 816-825

48. Pathuri P, Nguyen ET, Svard SG, Luecke H (2007) Apo and Calcium-bound Crystal Structures of Alpha-11 Giardin, an Unusual Annexin from Giardia lamblia. J. Mol. Biol. 368: 493-508

49. Pathuri P, Nguyen ET, Ozorowski G, Svärd SG, Luecke H (2009) Apo and calcium-bound crystal structures of cytoskeletal protein alpha-14 giardin (annexin E1) from the intestinal protozoan parasite Giardia lamblia. J. Mol. Biol. 385: 1098-1112

50. Hofmann A (2004) Annexins in the plant kingdom - perspectives and potentials. Annexins 1: 5161

51. Dabitz N, Hu NJ, Yusof AM, Tranter N, Winter A, Daley M, Zschörnig O, Brisson A, Hofmann A (2005) Structural determinants for plant annexin-membrane interactions. Biochemistry 44: 1629216300

52. Hu N, Yusof A, Winter A, Osman A, Reeve A, Hofmann A (2008) The crystal structure of calciumbound annexin Gh1 from Gossypium hirsutum indicates different mechanisms of membrane binding in plant and animal annexins. J. Biol. Chem. 283: 18314-18322 
53. Winter A, Yusof AM, Gao E, Yan HL, Hofmann A (2006) Biochemical characterization of annexin B1 from Cysticercus cellulosae. FEBS J. 273: 3238-3247

54. Römisch J, Heimburger N (1990) Purification and characterization of six annexins from human placenta. Biol. Chem. Hoppe Seyler 371: 383-388

55. Weiland M, Palm J, Griffiths W, McCaffery J, Svärd S (2003) Characterisation of alpha-1 giardin: an immunodominant Giardia lamblia annexin with glycosaminoglycan-binding activity. Int. J. Parasitol. 33: 1341-1351

56. Capila I, Hernaiz M, Mo Y, Mealy T, Campos B, Dedman J, Linhardt R, Seaton B (2001) Annexin V-heparin oligosaccharide complex suggests heparan sulfate-mediated assembly on cell surface. Structure 9: 57-64

57. Hofmann A, Osman A, Leow CY, Driguez P, Jones M (2010) Parasite annexins - new molecules with potential for drug and vaccine development. submitted:

58. Kaneko N, Ago H, Matsuda R, Inagaki E, Miyano M (1997) Crystal structure of annexin V with its ligand K-201 as a calcium channel activity inhibitor. J. Mol. Biol. 274: 16-20

59. Hofmann A, Escherich A, Lewit-Bentley A, Benz J, Raguenes-Nicol C, Russo-Marie F, Gerke V, Moroder L, Huber R (1998) Interactions of benzodiazepine derivatives with annexins. J. Biol. Chem. 273: $2885-2294$

60. Parente L, Solito E (2004) Annexin 1: more than an anti-phospholipase protein. Inflamm. Res. 53: $125-132$

61. Meers P, Mealy T, Pavlotsky N, Tauber Al (1992) Annexin I-mediated vesicular aggregation: mechanism and role in human neutrophils. Biochemistry 31: 6372-6382

62. Bitto E, Li M, Tikhonov AM, Schlossman ML, Cho W (2000) Mechanism of annexin I-mediated membrane aggregation. Biochemistry 39: 13469-13477

63. Streicher WW, Lopez MM, Makhatadze GI (2009) Annexin I and Annexin II N-Terminal Peptides Binding to S100 Protein Family Members: Specificity and Thermodynamic Characterization. Biochemistry 48: 2788-2798

64. Hu N, Bradshaw J, Lauter H, Buckingham J, Solito E, Hofmann A (2008) Membrane-induced folding and structure of membrane-bound annexin A1 N-terminal peptides - Implications for annexin-induced membrane aggregation. Biophys. J. 94: 1773-1781

65. Burgoyne R (2007) Neuronal calcium sensor proteins: generating diversity in neuronal Ca2+ signalling. Nat. Rev. Neurosci 8: 182-193

66. Genin A, Davis S, Meziane H, Doyere V, Jeromin A, Roder J, Mallet J, Laroche S (2001) Regulated expression of the neuronal calcium sensor-1 gene during long-term potentiation in the dentate gyrus in vivo. Neuroscience 106: 571-577

67. Braunewell K, Brackmann M, Manahan-Vaughan D (2003) Group I mGlu receptors regulate the 
expression of the neuronal calcium sensor protein VILIP-1 in vitro and in vivo: implications for mGlu receptor-dependent hippocampal plasticity?. Neuropharmacology 44: 707-715

68. Kabbani N, Negyessy L, Lin R, Goldman-Rakic P, Levenson R (2002) Interaction with neuronal calcium sensor NCS-1 mediates desensitization of the D2 dopamine receptor. J. Neurosci. 22: 84768486

69. Bahi N, Friocourt G, Carrié A, Graham ME, Weiss JL, Chafey P, Fauchereau F, Burgoyne RD, Chelly J (2003) IL1 receptor accessory protein like, a protein involved in X-linked mental retardation, interacts with Neuronal Calcium Sensor-1 and regulates exocytosis. Hum. Mol. Genet. 12: $1415-1425$

70. Cheng HM, Pitcher GM, Laviolette SR, Whishaw IQ, Tong KI, Kockeritz LK, Wada T, Joza NA, Crackower M, Goncalves J et al. (2002) DREAM is a critical transcriptional repressor for pain modulation. Cell 108: 31-43

71. Mahloogi H, Gonzalez-Guerrico AM, Lopez De Cicco R, Bassi DE, Goodrow T, Braunewell KH, Klein-Szanto AJ (2003) Overexpression of the calcium sensor visinin-like protein-1 leads to a CAMPmediated decrease of in vivo and in vitro growth and invasiveness of squamous cell carcinoma cells. Cancer Res. 63: 4997-5004

72. Heo WD, Inoue T, Park WS, Kim ML, Park BO, Wandless TJ, Meyer T $(2006)$ PI $(3,4,5) P 3$ and $\mathrm{PI}(4,5) \mathrm{P} 2$ lipids target proteins with polybasic clusters to the plasma membrane. Science 314 : 1458-1461

73. Czech MP (2000) PIP2 and PIP3: complex roles at the cell surface. Cell 100: 603-606

74. Tang K, Hofmann A, Freund C, Danker K (2009) The cytoplasmic tail of the alpha1 integrin subunit associates with phosphoinositides. submitted:

75. O'Callaghan DW, Haynes LP, Burgoyne RD (2005) High-affinity interaction of the N-terminal myristoylation motif of the neuronal calcium sensor protein hippocalcin with phosphatidylinositol 4,5-bisphosphate. Biochem. J. 391: 231-238

76. Braunewell KH, Brackmann M, Hofmann A (2006) VILIP-1, A novel regulator of the guanylate cyclase transduction system in neurons. Calcium Binding Proteins 1: 12-15

77. Brackmann M, Hofmann A, Braunewell KH (2006) Structure, function and expression of members of the vilip (visinin-like protein) subfamily of neuronal calcium sensor proteins. In: Philippov P, Koch K (ed) Neuronal calcium sensor proteins. Nova Science Publisher.

78. Braunewell K, Paul B, Altarche-Xifro W, Noack C, Lange K, Hofmann A (2010) Interactions of Visinin-like Proteins with Phospho-inositides. Aust. J. Chem. 63: 350-356

79. Krylov D, Hurley J (2001) Identification of Proximate Regions in a Complex of Retinal Guanylyl Cyclase 1 and Guanylyl Cyclase-activating Protein-1 by a Novel Mass Spectrometry-based Method. J. Biol. Chem 276: 30648-30654 
80. Chen K, Wang L, Chang L (2009) Regulatory elements and functional implication for the formation of dimeric visinin-like protein-1. J. Pep. Sci. 15: 89-94

81. McArdle B, Hofmann A (2008) Coronin structure and implications. In: Clemen C, Eichinger L, Rybakin V (ed) The coronin family of proteins. Landes Bioscience, Austin, TX.

82. Hudson AM, Cooley L (2008) Phylogenetic, structural and functional relationships between WD- and Kelch-repeat proteins. Subcell. Biochem. 48: 6-19

83. Smith TF (2008) Diversity of WD-repeat proteins. Subcell. Biochem. 48: 20-30

84. Smith TF, Gaitatzes C, Saxena K, Neer EJ (1999) The WD repeat: a common architecture for diverse functions. Trends Biochem Sci 24: 181-185

85. Li D, Roberts R (2001) WD-repeat proteins: structure characteristics, biological function, and their involvement in human diseases. Cell. Mol. Life Sci. 58: 2085-2097

86. Appleton B, Wu P, Wiesmann C (2006) The crystal structure of murine coronin-1: a regulator of actin cytoskeletal dynamics in lymphocytes. Structure 14: 87-96

87. de Hostos EL (1999) The coronin family of actin-associated proteins. Trends Cell. Biol. 9: 345350

88. Rybakin V, Clemen C (2005) Coronin proteins as multifunctional regulators of the cytoskeleton and membrane trafficking. BioEssays 27: 625-632

89. Rosentreter A, Hofmann A, Xavier CP, Stumpf M, Noegel AA, Clemen CS (2007) Coronin 3 involvement in F-actin-dependent processes at the cell cortex. Exp. Cell Res. 313: 878-895

90. Gatfield J, Albrecht I, Zanolari B, Steinmetz M, Pieters J (2005) Association of the leukocyte plasma membrane with the actin cytoskeleton through coiled coil-mediated trimeric coronin 1 molecules. Mol. Biol. Cell 16: 2786-2798

91. Gandhi M, Goode B (2008) Coronin: the double-edged sword of actin dynamics. In: Clemen C, Eichinger $L$, Rybakin $V($ ed) The coronin family of proteins. Landes Bioscience, Georgetown, TX.

92. Xavier C, Rosentreter A, Reimann J, Cornfine S, Linder S, van Vliet V, Hofmann A, Morgan RO, Fernandez M, Stumpf M et al. (2009) Structural and functional diversity of novel coronin-1C (CRN2) isoforms. J. Mol. Biol. 393: 287-299

93. Cai L, Holoweckyj N, Schaller M, Bear J (2005) Phosphorylation of coronin 1B by protein kinase C regulates interaction with Arp2/3 and cell motility. J. Biol. Chem 280: 31913-31923

94. Spoerl Z, Stumpf M, Noegel AA, Hasse A (2002) Oligomerization, F-actin Interaction, and Membrane Association of the Ubiquitous Mammalian Coronin 3 Are Mediated by Its Carboxyl Terminus. J. Biol. Chem. 277: 48858-48867

95. Schutt CE, Lindberg U (2000) The new architectonics: an invitation to structural biology. Anatom. Rec. 261: 198-216 
96. DeLano W (2002) The PyMOL Molecular Graphics System. http://www.pymol.org 
Figure 1

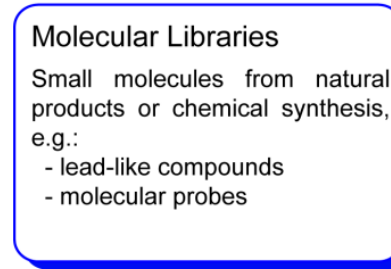

Structural Biology

Characterisation of molecular

structure and interactions using

methods in molecular cloning,

protein expression, and structure

determination, e.g.

- X-ray cystallography

Figure 1: Conceptual layout of the Structural Chemistry Program.

Molecular System and Protein Target

Parasitic Diseases

- Parasite-specific enzymes

- Proteins in host-pathogen

interactions

Neurological Diseases

- Visinin-like proteins

- Coronins

Molecular insight into

structure-function relationships
Molecular basis of disease

Towards development of novel drugs/therapeutics 


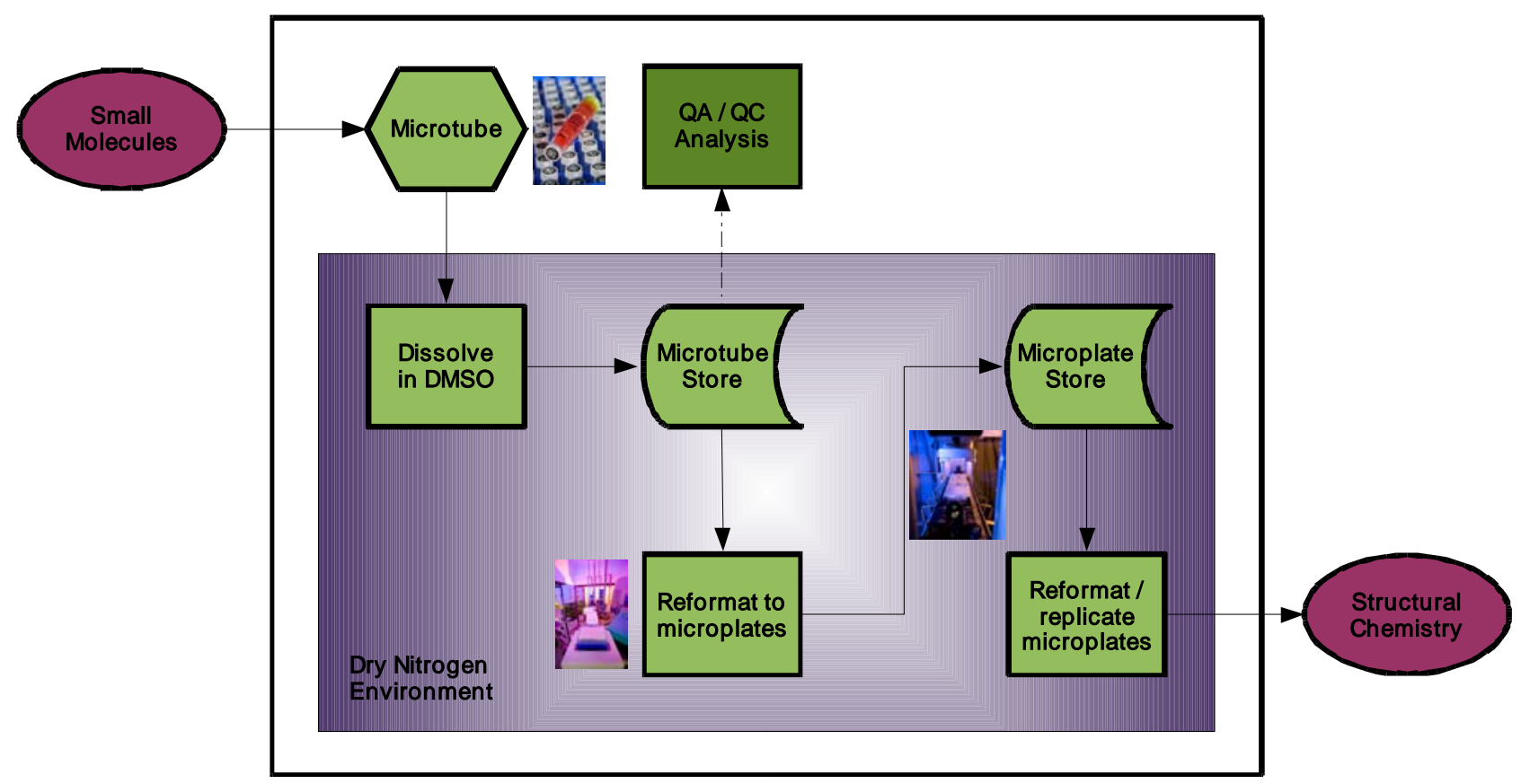

Figure 2: Schematic infrastructure layout of the Queensland Compound Library ( $Q C L)$. The $Q C L$ currently maintains 350,000 pure compounds as stock solutions in DMSO, stored in four microtube

stores. To facilitate rapid replication of this amount of samples, subsets have been prepared in 96 and 384 well plate format. Capacity for over 4,300 microtitre plates exists in the two plate stores. Since commencing operations in 2008 , over 3,500 racks of microtubes have been reformatted into 384 well 'working' plates from which 3,000 assay ready plates have been prepared. This equates to in excess of 1.1 million samples (at a rate of ca. 50,000 samples per month) being prepared for some type of biological evaluation or profiling. 
Figure 3

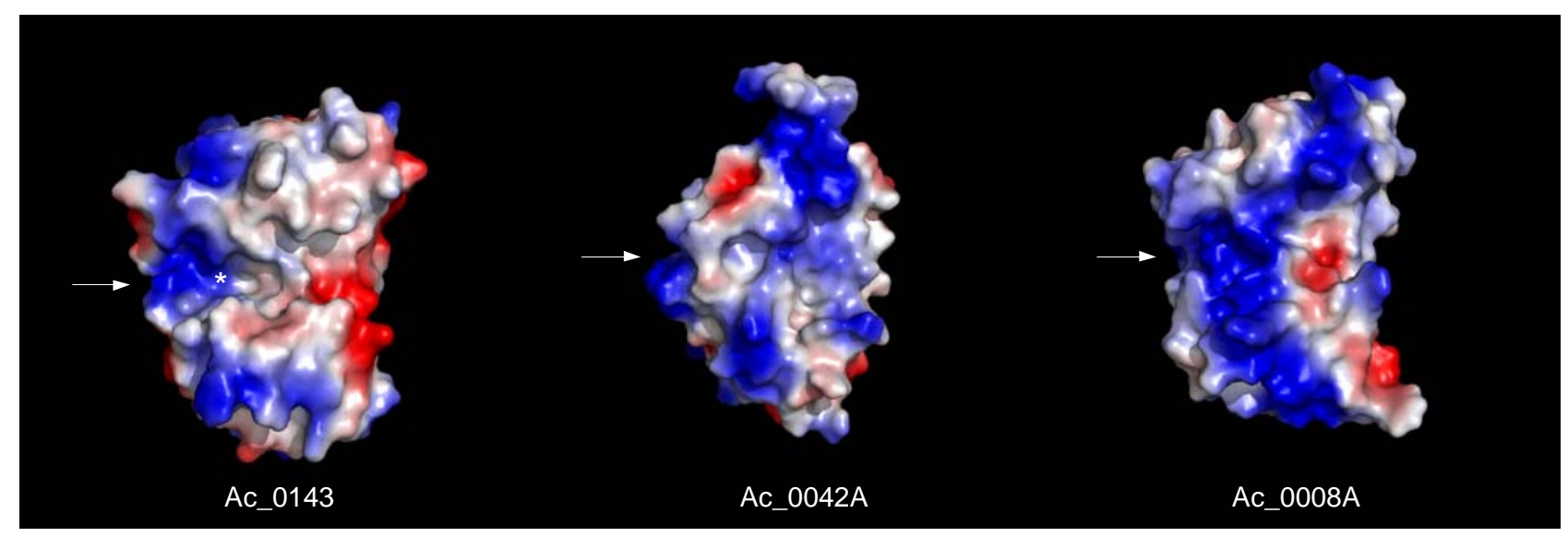

Figure 3: Models of representatives of the proposed three groups of Activation-associated secreted Proteins, Ac_0143 (Group 1), Ac_0042A (Group 2) and Ac_0008A (Group 3) from A. caninum. The models were obtained by comparative modelling using the structure of Na-ASP-2 [42] as template. The models are structurally aligned, and the view shown is the same for all three proteins. The colour code on the surface indicates the electrostatic potential (blue: basic; red: acidic). The figure was prepared with PyMOL [96].

The shape difference of Group 2 compared to Groups 1 and 3 is clearly visible. Furthermore, an equatorial groove (indicated by an arrow) is evident in all groups, but most pronounced in Group 1 ASPs. Additionally, in Group 1 ASPs, two conserved histidine side chains as well as residues close by in this groove adopt a conformation that is reminiscent of a catalytic site (indicated by '*'). 